\title{
Composite material design of two-dimensional structures using the homogenization design method
}

\author{
D. Fujiii ${ }^{*} \dagger$, B. C. Chen and N. Kikuchi \\ Department of Mechanical Engineering and Applied Mechanics, University of Michigan, Ann Arbor, \\ Michigan 48109-2125, U.S.A.
}

\begin{abstract}
SUMMARY
Composite materials of two-dimensional structures are designed using the homogenization design method. The composite material is made of two or three different material phases. Designing the composite material consists of finding a distribution of material phases that minimizes the mean compliance of the macrostructure subject to volume fraction constraints of the constituent phases, within a unit cell of periodic microstructures. At the start of the computational solution, the material distribution of the microstructure is represented as a pure mixture of the constituent phases. As the iteration procedure unfolds, the component phases separate themselves out to form distinctive interfaces. The effective material properties of the artificially mixed materials are defined by the interpolation of the constituents. The optimization problem is solved using the sequential linear programming method. Both the macrostructure and the microstructures are analysed using the finite element method in each iteration step. Several examples of optimal topology design of composite material are presented to demonstrate the validity of the present numerical algorithm. Copyright (C) 2001 John Wiley \& Sons, Ltd.
\end{abstract}

KEY WORDS: composite material; homogenization method; topology optimization; microstructures; numerical algorithm

\section{INTRODUCTION}

The homogenization design method (HDM) has been proposed by Bendsøe and Kikuchi [1] and Suzuki and Kikuchi [2] in order to obtain the optimum shape and topology of the continuum structure. In this method, the boundary shape optimization problem is converted into the material optimization problem in the extended design domain based on the approach of Murat and Tartar [3]. It is assumed that the extended design domain is composed of porous materials, and the optimum topology and shape of the macrostructure are obtained by optimizing the size and the direction of the material holes in the microstructure (see Figure 1).

\footnotetext{
*Correspondence to: D. Fujii, Department of Environmental and Ocean Engineering, The University of Tokyo. 7-3-1 Hongo, Bunkyo-ku, Tokyo 113-8656, Japan

†E-mail: dfujii@nasl.t.u-tokyo.ac.jp
}

Contract/grant Sponsor. Steel Material Club Corporation Aggregate, Japan

Copyright (C) 2001 John Wiley \& Sons, Ltd.

Received 4 February 1999 Revised 22 September 1999 


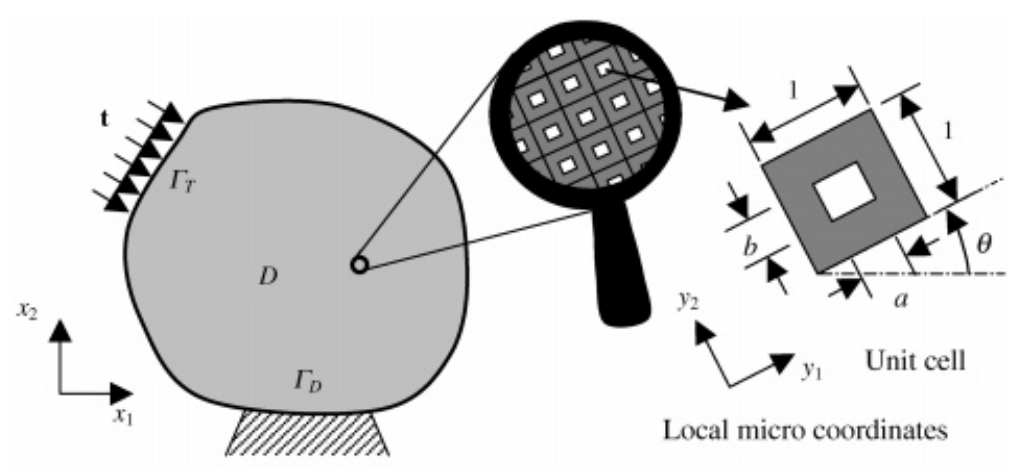

Global macro coordinates

Figure 1. A two-dimensional structure with porous microstructures.

In this work, there is no need to limit the material microstructure to a particular porous pattern, and it can be of any arbitrary shape and topology. In addition, it is also possible to design the material microstructure which realizes the optimum components of the elasticity tensor of the macrostructure, if the optimum components of that are already known. Several researchers have addressed the problem of designing the microstructure of the composite material which realizes the optimum elasticity constants of the macrostructure based on this idea. Sigmund et al. [4-7] and Fonseca [8] formulated this problem as an optimization problem by searching for the lightest microstructure with the prescribed thermoelastic properties specified as equality constraints. Swan and Arora [8] and Swan and Kosaka [10] designed inelastic composites to enhance stiffness and strength. Silva et al. [11, 12] and Gibiansky and Torquato [13, 14] designed the microstructure of piezocomposites with improved performance characteristics. Haslinger and Dvorak [15] treated this problem as an identification problem.

However, the loading and the boundary conditions of the macrostructure change the optimum characteristics of the macrostructure actually. In some cases, it is difficult to determine the optimum characteristics of the macrostructure. The simultaneous analysis of the macrostructure and the microstructure based on the original idea of Bendsøe and Kikuchi [1] is necessary for such cases. Therefore, in this paper, it is assumed that the design domain is composed of the composite material with two or three material phases instead of the porous material, and the material layout of unit cell of the microstructure is chosen as the design object. In other words, in the present method, the topology of the unit cell is free to change to any pattern, while the microstructure pattern is parameterized as a square region with a sizeable rectangular hole in the original idea of Bendsøe and Kikuchi [1]. As an initial attempt, the simple problem in which the macrostructure is uniform is handled in this paper because the number of the design variables becomes enormous if the microstructures assume non-uniform distribution within the macroscopic design domain.

The present design procedure consists of finding a distribution of material phases that minimizes the mean compliance of the macrostructure subject to volume fraction constraints of the constituent phases, within a unit cell of periodic microstructures. Concretely, the material of each element of the unit cell with finite element discretization is assigned from two or three material phases. However, in this discretion optimization, the number of the combination becomes enormous, as the number of division of the finite element increases, and it is hopeless to solve the optimization problem using random search methods such as, genetic algorithms or simulated annealing methods [7]. 
Therefore, in this paper, the mixing rule used in the analysis of macrostructure (e.g. Bendsøe [16], Rozvany et al. [17], Mlejnek and Schirrmacher [18], Yang and Chuang [19]), is applied to the microstructure design as one can find in literature [4-15].

Although the mixing rules for two materials mixture involving linear elastic solid and void phases have been proposed, the mixing rule for three materials mixture is not found except for Sigmund and Torquato [7]. The three-material-phase design is more advantageous than the two-materialphase design when the temperature characteristics, permeability, lightening, etc. are considered. The mixing rule for two materials and void mixture proposed by Sigmund and Torquato [7] is composed of the classical Voigt mixing rule for two solid materials and the power law for void and solid ones. However, if we use the Voigt mixing rule, the mixture of the two material phases dose not separate in the final stage of the iteration procedure for the optimization, as Swan and Kosaka [20] has reported. Therefore, the new mixing rule of the three materials mixture based on the power laws is proposed in this paper.

It is well known that the checkerboarding material distribution is easily obtained if the underlying mixing rule based on density approach is employed [20,21]. In order to avoid the checkerboarding solutions, it is necessary to use the high-order finite element methods [7, 22, 23], or to use filtering methods [20, 24-27]. In this paper, the low-order finite element method for the analysis of the microstructure is used in order to save calculation time, and a novel filtering method, based on the concept of gravity, is proposed.

The optimization problem is solved using sequential linear programming. The method for relaxing the volume fraction constraints of the constituent phases in initial stage of the iterations is used in order to avoid the convergence to the local minima. Some examples of optimum topology design of composite materials for two-dimensional structures are presented to demonstrate the validity of the proposed filtering method and the present numerical algorithm.

The paper is organized in the following way. In Section 2 we describe the formulation of the optimization problem and its discretization. The topology optimization procedure is described in Section 3. In Section 4, the checkerboarding problem is discussed, and a new filtering method is presented. The performance of the proposed filtering method is demonstrated with an example. In Section 5, the effectiveness of the present algorithm is shown by some examples which have different boundary and loading conditions.

\section{FORMULATION OF THE OPTIMIZATION PROBLEM}

\subsection{Optimization problem in the homogenization design method}

In the homogenization design method (HDM) proposed by Bendsøe and Kikuchi [1], it is assumed that the extended domain is composed of the porous material, and the size and the direction of the holes of the microstructure which minimize the mean compliance of the macrostructure are obtained. This optimization problem can be expanded in the more general form as follows:

$$
\begin{aligned}
& \max _{\left\{D_{i j}^{\mathrm{H}}\right\}}\left[\min _{\mathbf{v}_{0} \in \mathrm{K}} \Pi\left(\mathbf{v}_{0}\right)\right], \quad \mathbf{K}=\left\{\mathbf{v}_{0} \mid \mathbf{v}_{0}=\mathbf{0} \text { on } \Gamma_{D}\right\} \\
& \Pi\left(\mathbf{v}_{0}\right)=\frac{1}{2} \int_{D} \boldsymbol{\varepsilon}\left(\mathbf{v}_{0}\right)^{\mathrm{T}} \mathbf{D}^{\mathrm{H}} \boldsymbol{\varepsilon}\left(\mathbf{v}_{0}\right) \mathrm{d} D-\int_{\Gamma_{\mathrm{T}}} \mathbf{v}_{0}^{\mathrm{T}} \mathbf{t} \mathrm{d} \Gamma
\end{aligned}
$$




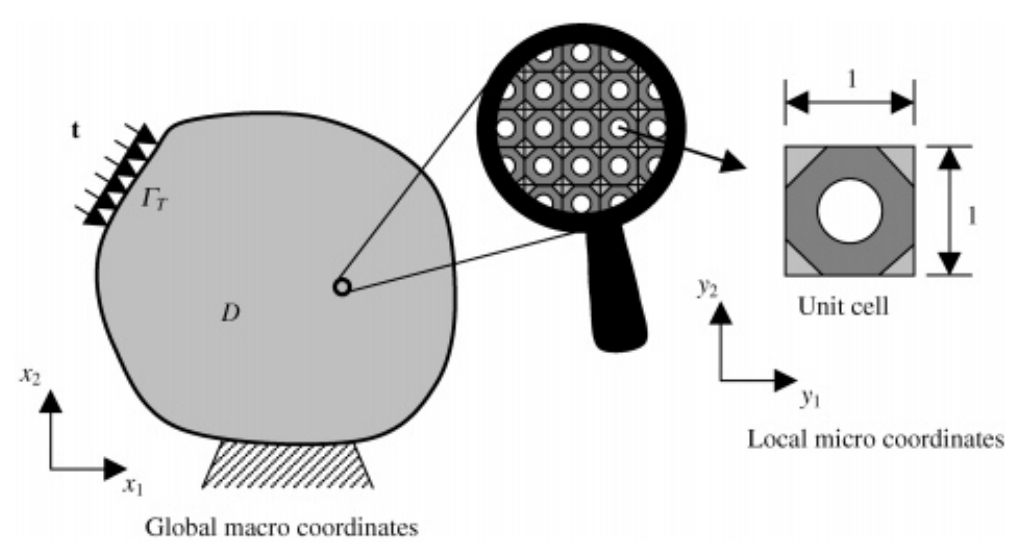

Figure 2. A two-dimensional structure with composite material microstructures.

where $\Pi$ is the total potential energy, $\mathbf{v}_{0}$ the displacement vector of the macrostructure, $\varepsilon$ the strain tensor, $\mathbf{D}^{\mathrm{H}}$ the homogenized elasticity matrix, $D_{i j}^{\mathrm{H}}$ the components of $\mathbf{D}^{\mathrm{H}}$, and $\mathbf{t}$ the boundary traction vector (see Figure 1). Here, it is assumed that the body force can be neglected.

The optimization problem in Equation (1) searches the optimal components $D_{i j}^{\mathrm{H}}$ which maximize the minimum potential energy of the macrostructure. In the problem in which loading condition is given, Equation (1) becomes the minimization problem of the compliance. On the other hand, for the problem in which enforcement displacement is given, it becomes the maximization problem of the compliance. In the problem in search of the topology of the macrostructure, $\mathbf{D}^{\mathrm{H}}$ becomes the function of the size and direction of the hole in the porous microstructure, namely $\mathbf{D}^{\mathrm{H}}(a, b, \theta)$.

In this paper, the microstructure of the extended design domain $D$ is composed of the uniform periodic arrangement of the unit cell as shown in Figure 2, and the unit cell is made by the layout of 2 or 3 kinds of material phases. In this problem, $\mathbf{D}^{\mathrm{H}}$ is decided by the layout of material phases in the unit cell. Therefore, the layout of material phases in the unit cell is the design object of this problem.

\subsection{Homogenized minimum potential energy}

The homogenized elasticity matrix $\mathbf{D}^{\mathrm{H}}$ is obtained by the homogenization method [28]. In this method, two kinds of co-ordinate systems, the global macro-co-ordinates $\mathbf{x}$ and the local micro-coordinates $\mathbf{y}$, are used, as shown in Figure 2. The relationship $\mathbf{y}=\mathbf{x} / \varepsilon$ is established between the two co-ordinate systems, where $\varepsilon$ is the representative length of the microstructure, and $\varepsilon$ is assumed to be small enough compared with the dimension of the macrostructure. And the microstructure is supposed to have the periodicity. In this case, the displacement field can be approximated by an asymptotic expansion

$$
\mathbf{v}^{\varepsilon}(\mathbf{x})=\mathbf{v}(\mathbf{x}, \mathbf{y}) \approx \mathbf{v}_{0}(\mathbf{x})+\varepsilon \mathbf{v}_{1}(\mathbf{x}, \mathbf{y})
$$

where the superscript $\varepsilon$ indicates the existence of the microstructure, $\mathbf{v}_{0}$ is the average displacement of the unit cell, $\mathbf{v}_{1}$ is the first-order variation from the average displacement.

The strain and stress tensor also can be approximated by

$$
\varepsilon\left(\mathbf{v}^{\varepsilon}\right)=\partial_{\mathrm{x}} \mathbf{v}^{\varepsilon} \approx \partial_{\mathrm{x}} \mathbf{v}_{0}+\partial_{\mathrm{y}} \mathbf{v}_{1}=\varepsilon_{0}+\varepsilon_{1}
$$


where $\varepsilon_{0}$ is the strain due to the average displacement, $\varepsilon_{1}$ is the strain due to the first deviation of the displacement from the average displacement of the unit cell, and $\partial_{\mathbf{x}}, \partial_{\mathbf{y}}$ are the differential operators which are expressed in the two dimensional problem as follows:

$$
\boldsymbol{\partial}_{\mathrm{x}}=\left[\begin{array}{cc}
\partial / \partial x_{1} & 0 \\
0 & \partial / \partial x_{2} \\
\partial / \partial x_{2} & \partial / \partial x_{1}
\end{array}\right], \quad \boldsymbol{\partial}_{\mathrm{y}}=\left[\begin{array}{cc}
\partial / \partial y_{1} & 0 \\
0 & \partial / \partial y_{2} \\
\partial / \partial y_{2} & \partial / \partial y_{1}
\end{array}\right]
$$

The stress is defined by

$$
\boldsymbol{\sigma}\left(\mathbf{v}^{\varepsilon}\right)=\mathbf{D} \boldsymbol{\varepsilon}\left(\mathbf{v}^{\varepsilon}\right) \approx \mathbf{D}\left(\partial_{\mathrm{x}} \mathbf{v}_{0}+\partial_{\mathrm{y}} \mathbf{v}_{1}\right)
$$

where $\mathbf{D}$ is the elasticity matrix.

Because $\mathbf{v}_{1}$ is the first-order variation from the average displacement, it is possible that $\mathbf{v}_{1}$ is considered to be proportional for the strain $\varepsilon_{0}$ :

$$
\mathbf{v}_{1}=-\chi(\mathbf{y}) \varepsilon_{0}(\mathbf{x})=-\chi(\mathbf{y}) \partial_{\mathrm{x}} \mathbf{v}_{0}(\mathbf{x})
$$

where $\chi$ is the $2 \times 3$ matrix in the two-dimensional problem, and it indicates a kind of proportionality constant. $\chi$ is called the characteristic displacement function of the microstructure, because it is the displacement for the unit strain of the macrostructure.

Substituting Equation (7) into Equations (4) and (6), the total potential energy is expressed as

$$
\Pi\left(\mathbf{v}^{\varepsilon}\right)=\frac{1}{2} \int_{D^{\varepsilon}}\left(\partial_{\mathrm{x}} \mathbf{v}_{0}\right)^{\mathrm{T}}\left(\mathbf{I}-\partial_{\mathrm{y}} \chi\right)^{\mathrm{T}} \mathbf{D}\left(\mathbf{I}-\partial_{\mathrm{y}} \boldsymbol{\chi}\right)\left(\partial_{\mathrm{x}} \mathbf{v}_{0}\right) \mathrm{d} D^{\varepsilon}-\int_{\Gamma_{\mathrm{T}}} \mathbf{v}_{0}^{T} \mathbf{t} \mathrm{d} \Gamma
$$

The following equation

$$
\lim _{\varepsilon \rightarrow 0} \int_{D^{\varepsilon}} \Phi^{\varepsilon}(\mathbf{x}) \mathrm{d} D^{\varepsilon}=\int_{D}\left(\frac{1}{|Y|} \int_{Y} \Phi(\mathbf{x}, \mathbf{y}) \mathrm{d} Y\right) \mathrm{d} D
$$

is considered, and by $\varepsilon \rightarrow 0$

$$
\Pi\left(\mathbf{v}^{\varepsilon}\right)=\frac{1}{2} \int_{D}\left(\partial_{\mathrm{x}} \mathbf{v}_{0}\right)^{\mathrm{T}} \frac{1}{|Y|} \int_{Y}\left(\mathbf{I}-\boldsymbol{\partial}_{\mathrm{y}} \boldsymbol{\chi}\right)^{\mathrm{T}} \mathbf{D}\left(\mathbf{I}-\boldsymbol{\partial}_{\mathrm{y}} \boldsymbol{\chi}\right) \mathrm{d} Y\left(\boldsymbol{\partial}_{\mathrm{x}} \mathbf{v}_{0}\right) \mathrm{d} D-\int_{\Gamma_{T}} \mathbf{v}_{0}^{\mathrm{T}} \mathbf{t} \mathrm{d} \Gamma
$$

is obtained where $\mathbf{I}$ is the identity matrix, and $|Y|$ the area of unit cell. We define

$$
\mathbf{D}^{Y}=\frac{1}{|Y|} \int_{Y}\left(\mathbf{I}-\partial_{\mathrm{y}} \boldsymbol{\chi}\right)^{\mathrm{T}} \mathbf{D}\left(\mathbf{I}-\boldsymbol{\partial}_{\mathrm{y}} \boldsymbol{\chi}\right) \mathrm{d} Y
$$

then Equation (10) is expressed as follows:

$$
\Pi\left(\mathbf{v}^{\varepsilon}\right)=\frac{1}{2} \int_{D}\left(\partial_{\mathrm{x}} \mathbf{v}_{0}\right)^{\mathrm{T}} \mathbf{D}^{Y}\left(\partial_{\mathrm{x}} \mathbf{v}_{0}\right) \mathrm{d} D-\int_{\Gamma_{T}} \mathbf{v}_{0}^{\mathrm{T}} \mathbf{t} \mathrm{d} \Gamma
$$

From Equation (12), the minimum potential energy is obtained as follows:

$$
\min _{\mathbf{v}^{\varepsilon}}\left[\Pi\left(\mathbf{v}^{\varepsilon}\right)\right] \approx \min _{\mathbf{v}_{0}, \chi}\left[\Pi\left(\mathbf{v}_{0}\right)\right]=\Pi^{\mathrm{H}}\left(\mathbf{u}_{0}\right)=\min _{\mathbf{v}_{0}}\left[\frac{1}{2} \int_{D}\left(\partial_{\mathrm{x}} \mathbf{v}_{0}\right)^{\mathrm{T}} \mathbf{D}^{\mathrm{H}}\left(\boldsymbol{\partial}_{\mathrm{x}} \mathbf{v}_{0}\right) \mathrm{d} D-\int_{\Gamma_{T}} \mathbf{v}_{0}^{\mathrm{T}} \mathbf{t} \mathrm{d} \Gamma\right]
$$


where $\Pi^{\mathrm{H}}\left(\mathbf{u}_{0}\right)$ is the homogenized minimum potential energy, and $\mathbf{D}^{\mathrm{H}}$ is the homogenized elasticity matrix defined as follows:

$$
\mathbf{D}^{\mathrm{H}}=\min _{\substack{\chi \\ \text { Periodic }}} \mathbf{D}^{Y}=\min _{\substack{\boldsymbol{\chi} \\ \text { Periodic }}}\left[\frac{1}{|Y|} \int_{Y}\left(\mathbf{I}-\boldsymbol{\partial}_{y} \boldsymbol{\chi}\right)^{\mathrm{T}} \mathbf{D}\left(\mathbf{I}-\boldsymbol{\partial}_{y} \boldsymbol{\chi}\right) \mathrm{d} Y\right]
$$

Here, $\Pi\left(\mathbf{v}_{0}\right)$ in Equation (13) is equal to Equation (2).

\subsection{Discretization for the macrostructure}

In order to solve the optimization problem defined by Equation (1), the minimum potential energy defined by Equation (13) is discretized using the finite element method.

The displacement field in an element is expressed using the nodal displacements of the element as follows:

$$
\mathbf{v}_{0}=\mathbf{N}_{0} \mathbf{d e}_{\mathrm{e}}^{\mathrm{L}}
$$

where $\mathbf{N}_{0}$ is the shape function, and $\mathbf{d}_{\mathrm{e}}^{\mathrm{L}}$ is the nodal displacement vector with respect to the local co-ordinate system. From Equation (15),

$$
\boldsymbol{\varepsilon}_{0}=\partial_{\mathrm{x}} \mathbf{v}_{0}=\mathbf{B}_{0} \mathbf{d}_{\mathrm{e}}^{\mathrm{L}}
$$

is obtained, where $\mathbf{B}_{0}$ is the strain-displacement matrix. And the potential energy for an element is as follows:

$$
\Pi_{\mathrm{e}}=\frac{1}{2} \mathbf{d}_{\mathrm{e}}^{\mathrm{L}^{\mathrm{T}}} \int_{D_{\mathrm{e}}} \mathbf{B}_{0}^{\mathrm{T}} \mathbf{D}^{\mathrm{H}} \mathbf{B}_{0} \mathbf{d} D_{\mathrm{e}} \mathbf{d}_{\mathrm{e}}^{\mathrm{L}}-\mathbf{d}_{\mathrm{e}}^{\mathrm{L}^{\mathrm{T}}} \int_{\Gamma_{T_{\mathrm{e}}}} \mathbf{N}_{0}^{\mathrm{T}} \mathbf{t} \mathrm{d} \Gamma_{\mathrm{e}}
$$

The displacement vector $\mathbf{d}_{\mathrm{e}}^{\mathrm{L}}$ is transformed to $\mathbf{d}_{\mathrm{e}}$ with respect to the global co-ordinate system by

$$
\mathbf{d}_{\mathrm{e}}^{\mathrm{L}}=\mathbf{T}_{\mathrm{e}} \mathbf{d}_{\mathrm{e}}
$$

where $\mathbf{T}_{\mathrm{e}}$ is the coordinate transformation matrix. Substitute Equation (18) into Equation (17), then

$$
\Pi_{\mathrm{e}}=\frac{1}{2} \mathbf{d}_{\mathrm{e}}^{\mathrm{T}} \mathbf{T}_{\mathrm{e}}^{\mathrm{T}} \int_{D_{\mathrm{e}}} \mathbf{B}_{0}^{\mathrm{T}} \mathbf{D}^{\mathrm{H}} \mathbf{B}_{0} \mathrm{~d} D_{\mathrm{e}} \mathbf{T}_{\mathrm{e}} \mathbf{d}_{\mathrm{e}}-\mathbf{d}_{\mathrm{e}} \mathbf{T}_{\mathrm{e}}^{\mathrm{T}} \int_{\Gamma_{T \mathrm{e}}} \mathbf{N}_{0}^{\mathrm{T}} \mathbf{t} \mathrm{d} \Gamma_{\mathrm{e}}
$$

When the total potential energy is minimum, $\delta \Pi_{\mathrm{e}}=0$ is consistent, and

$$
\mathbf{K}_{0 \mathrm{e}} \mathbf{d}_{\mathrm{e}}=\mathbf{f}_{\mathrm{e}}
$$

where

$$
\begin{aligned}
\mathbf{K}_{0 \mathrm{e}} & =\mathbf{T}_{\mathrm{e}}^{\mathrm{T}} \int_{D_{\mathrm{e}}} \mathbf{B}_{0}^{\mathrm{T}} \mathbf{D}^{\mathrm{H}} \mathbf{B}_{0} \mathrm{~d} D_{\mathrm{e}} \mathbf{T}_{\mathrm{e}} \\
\mathbf{f}_{\mathrm{e}} & =\mathbf{T}_{\mathrm{e}}^{\mathrm{T}} \int_{\Gamma_{T \mathrm{e}}} \mathbf{N}_{0}^{\mathrm{T}} \mathbf{t} \mathrm{d} \Gamma_{\mathrm{e}}
\end{aligned}
$$




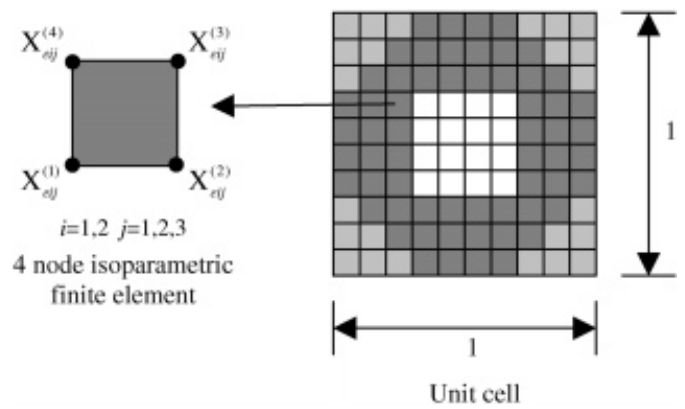

Figure 3. Discretization of a unit cell.

The following global stiffness equation is obtained by assembling the element stiffness equation defined by Equation (20) for all elements.

$$
\mathbf{K}_{0} \mathbf{d}=\mathbf{f}
$$

In the problem in which the loading condition is prescribed, Equation (23) is solved considering the geometrical boundary condition, and using this solution, the minimum potential energy is obtained as follows:

$$
\Pi^{\mathrm{H}}\left(\mathbf{u}_{0}\right)=\frac{1}{2} \mathbf{d}^{\mathrm{T}} \mathbf{K}_{0} \mathbf{d}-\mathbf{d}^{\mathrm{T}} \mathbf{f}=-\frac{1}{2} \mathbf{d}^{\mathrm{T}} \mathbf{K}_{0} \mathbf{d}
$$

Therefore, Equation (1) can be written as follows:

$$
\min _{\left\{D_{i j}^{\mathrm{H}}\right\}}\left[\mathbf{d}^{\mathrm{T}} \mathbf{K}_{0} \mathbf{d}\right]
$$

\subsection{Discretization for the microstructure}

The homogenized elasticity matrix $\mathbf{D}^{\mathrm{H}}$ defined by Equation (14) is also discretized by the finite element method. The unit cell is divided by the finite elements as shown in Figure 3. The characteristic displacement function $\chi$ and its strain $\partial_{y} \chi$ in an element is approximated using the nodal displacement function $\mathbf{X}_{\mathrm{e}}$ of the element as follows

$$
\begin{aligned}
\chi & =\mathbf{N}_{1} \mathbf{X}_{\mathrm{e}} \\
\partial_{y} \chi & =\mathbf{B}_{1} \mathbf{X}_{\mathrm{e}}
\end{aligned}
$$

where $\mathbf{N}_{1}$ and $\mathbf{B}_{1}$ are the shape function and the strain-displacement matrix for the microstructure, respectively. Using Equations (26) and (27), $\mathbf{D}^{Y}$ defined by Equation (11) is expressed as follows:

$$
\mathbf{D}^{Y}=\frac{1}{\left|Y_{\mathrm{e}}\right|} \int_{Y_{\mathrm{e}}}\left(\mathbf{I}-\mathbf{B}_{1} \mathbf{X}_{\mathrm{e}}\right)^{\mathrm{T}} \mathbf{D}\left(\mathbf{I}-\mathbf{B}_{1} \mathbf{X}_{\mathrm{e}}\right) \mathrm{d} Y_{\mathrm{e}}
$$

The necessary condition for the minimizing of Equation (28) is

$$
\delta \mathbf{X}_{\mathrm{e}}\left(\int_{Y_{\mathrm{e}}} \mathbf{B}_{1} \mathbf{D} \mathrm{d} Y_{\mathrm{e}}-\int_{Y_{\mathrm{e}}} \mathbf{B}_{1} \mathbf{D} \mathbf{B}_{1} \mathrm{~d} Y_{\mathrm{e}} \mathbf{X}_{\mathrm{e}}\right)=0
$$


From Equation (29), the following equation is obtained:

$$
\mathbf{K}_{1 \mathrm{e}} \mathbf{X}_{\mathrm{e}}=\mathbf{q}_{\mathrm{e}}
$$

where

$$
\begin{aligned}
\mathbf{K}_{1 \mathrm{e}} & =\int_{Y_{\mathrm{e}}} \mathbf{B}_{1} \mathbf{D} \mathbf{B}_{1} \mathrm{~d} Y_{\mathrm{e}} \\
\mathbf{q}_{\mathrm{e}} & =\int_{Y_{\mathrm{e}}} \mathbf{B}_{1} \mathbf{D} \mathrm{d} Y_{\mathrm{e}}
\end{aligned}
$$

The following equation is obtained by assembling Equation (30) for all elements in the unit cell.

$$
\mathbf{K}_{1} \mathbf{X}=\mathbf{q}
$$

Equation (33) is solved considering the periodicity condition of the unit cell, and using this solution, $\mathbf{D}^{\mathrm{H}}$ defined by Equation (14) is calculated by the following equation:

$$
\mathbf{D}^{\mathrm{H}}=\sum_{\text {elements }} \int_{Y_{\mathrm{e}}}\left(\mathbf{I}-\mathbf{B}_{1} \mathbf{X}_{\mathrm{e}}\right)^{\mathrm{T}} \mathbf{D}\left(\mathbf{I}-\mathbf{B}_{1} \mathbf{X}_{\mathrm{e}}\right) \mathrm{d} Y_{\mathrm{e}}
$$

\section{TOPOLOGY OPTIMIZATION PROCEDURE}

The optimization problem defined by Equations (1) and (2) in the discretized form is stated in Equations (25) and (34). The design object $\left\{D_{i j}^{\mathrm{H}}\right\}$ in Equation (25) is calculated by Equation (34). In this paper, the unit cell is composed of two- or three-phase material structures (see Figure 4). Therefore, the optimization problem consists of assigning the material phase to each element in the unit cell from two or three material phases. In other words, it is to find the topology of each material phase in the unit cell. Here, it is assumed that the material properties in each phase are linear elastic and isotropic.

\subsection{Constitutive mixing rules}

In this paper, the density approach [17-19] proposed for the topology optimization analysis of the macrostructure is adopted for the analysis of the microstructure. In the density approach, the solid material and void are mixed artificially using the power law. This mixing rule also can be applied to two kinds of solid materials.

It is known that if one uses the Voigt mixing rule (the parallel spring model, see Figure 5), the mixed material is not decomposed in the final solution, that is to say, intermediate density (gray scale) is remained in the topology solution. On the other hand, if we use the Reuss mixing rule (the serial spring model, see Figure 5), the mixed material is decomposed clearly. However, in the case of the mixture of solid material and void, the variation of the stiffness of the mixed material is extremely steep (it will be shown in Figure 6(b) later). The mixing rule with power law corresponds to the intermediate one between Voigt and Reuss mixing rules. As an alternative, the hybrid rule of the Voigt and Reuss mixing rules was proposed by Swan and Kosaka [20]. Though the hybrid rule is more effective for the elastoplastic problem, the power law is adopted in this paper because this rule is simpler than the hybrid rule for the three material phase problem. 


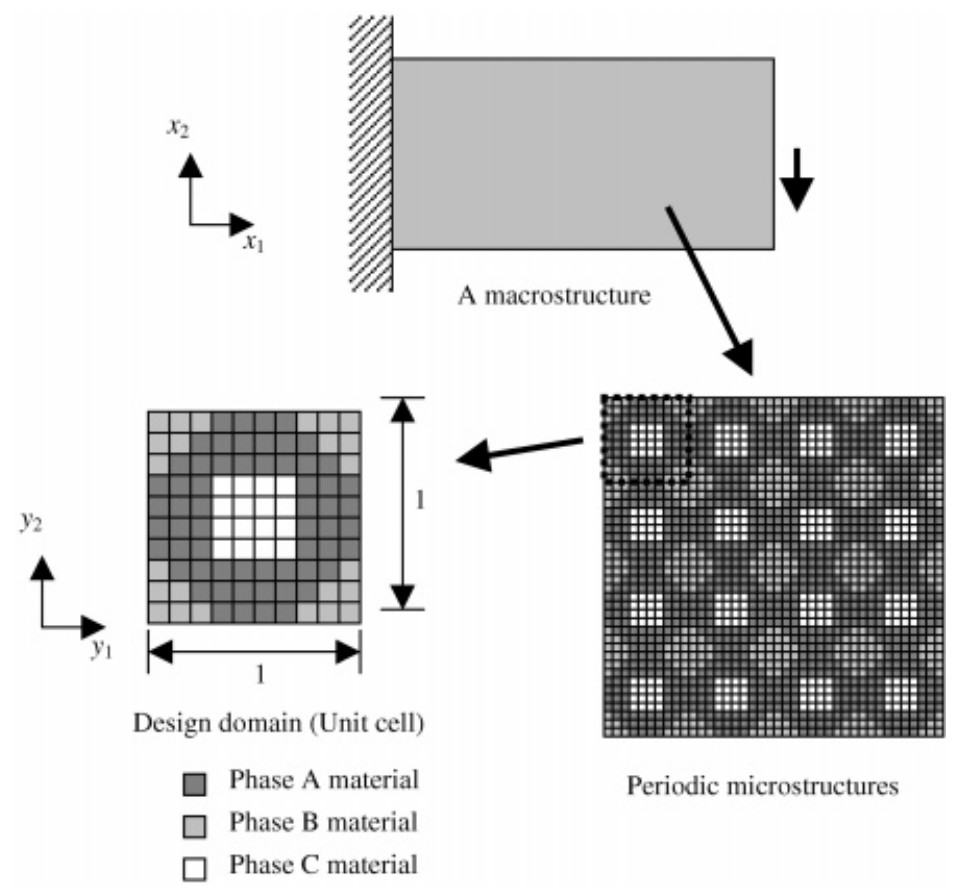

Figure 4. Design domain for topology optimization of composite material microstructures. Each square represents one finite element which consists of either phase $A, B$ or $C$.

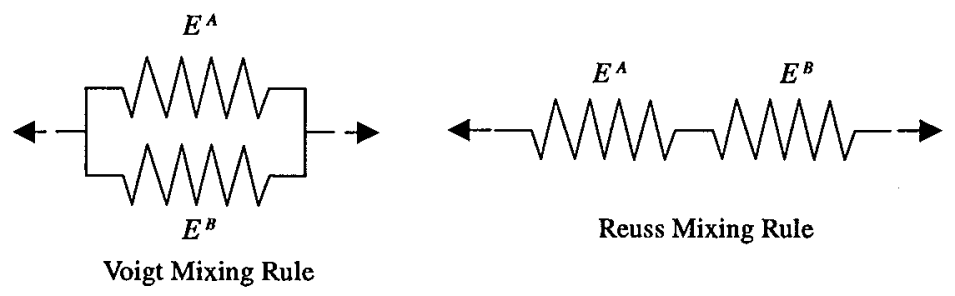

Figure 5. Voigt mixing rule and Reuss mixing rule, where $E^{\mathrm{A}}$ and $E^{\mathrm{B}}$ are the stiffness of $A$ and $B$ materials.

So far, we have addressed the mixing rule for two-phase material. However, in the design of composite material, three-phase material design is more advantageous when the temperature characteristics, permeabilities, lightening, ecology, etc. are considered. Sigmund and Torquato [7] addressed the design of materials using three kinds of materials, viz. two solid materials and void. They used the mixing rule which combines the power law and the Voigt mixing. However, as it has been mentioned above, it is easy to have overlapping between the constituent phases in the final solution when the Voigt mixing rule is used. Therefore, in this paper, instead of using the material rule based on the Voigt mixing, a new mixing rule, which used the combines power laws for three-phase material design is proposed. 

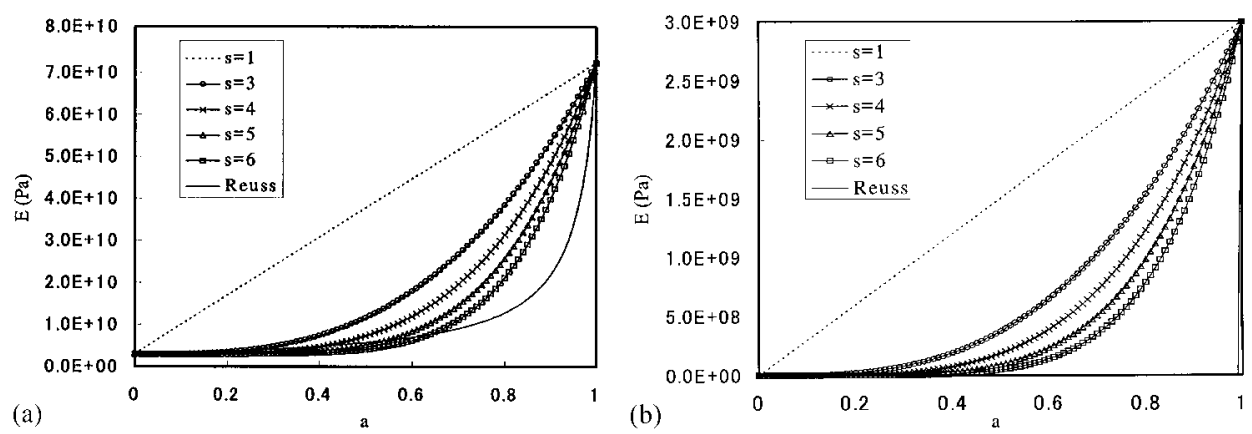

Figure 6. Relationship between $E$ and $a$ for the variation of $s$ in the case of: (a) $E^{\mathrm{A}}=72.4 \mathrm{GPa}, E^{\mathrm{B}}=3.0 \mathrm{GPa}$ and $b=1 ;(\mathrm{b}) E^{\mathrm{A}}=3.0 \mathrm{Gpa}, E^{\mathrm{B}}=100 \mathrm{~Pa}$ and $b=1$.

In considering the microstructures made of constituent phases $\mathrm{A}-\mathrm{C}$, the Young's moduli for each constituent phases are denoted by $E^{\mathrm{A}}, E^{\mathrm{B}}, E^{\mathrm{C}}$. In this case, the Young's modulus $E$ of the mixed material is defined using the power law as follows:

$$
E=r\left\{p E^{\mathrm{A}}+(1-p) E^{\mathrm{B}}\right\}+(1-r)\left\{q E^{\mathrm{C}}+(1-q) E^{\mathrm{B}}\right\}
$$

where $p, q$ and $r$ are defined by

$$
\begin{aligned}
& p=a^{s} \quad \text { if } E^{\mathrm{B}} \leqslant E^{\mathrm{A}} \\
& p=1-(1-a)^{s} \quad \text { if } E^{\mathrm{B}}>E^{\mathrm{A}} \\
& q=a^{s} \quad \text { if } E^{\mathrm{B}} \leqslant E^{\mathrm{C}} \\
& q=1-(1-a)^{s} \quad \text { if } E^{\mathrm{B}}>E^{\mathrm{C}} \\
& r=b^{s} \quad \text { if }\left\{q E^{\mathrm{C}}+(1-q) E^{\mathrm{B}}\right\} \leqslant\left\{p E^{\mathrm{A}}+(1-p) E^{\mathrm{B}}\right\} \\
& r=1-(1-b)^{s} \quad \text { if }\left\{q E^{\mathrm{C}}+(1-q) E^{\mathrm{B}}\right\}>\left\{p E^{\mathrm{A}}+(1-p) E^{\mathrm{B}}\right\}
\end{aligned}
$$

and $0 \leqslant a \leqslant 1$ and $0 \leqslant b \leqslant 1$. In addition, the power $s$ in Equations (36)-(41) is the factor used to penalize the intermediate properties. For a special case when $b=1$, the problem degenerates into a two-phase design composed of phases $\mathrm{A}$ and $\mathrm{B}$. As one can observe from above equations, if $a=1, b=1$ then $E=E^{\mathrm{A}}$; if $a=0$ then $E=E^{\mathrm{B}}$; and if $a=1, b=0$ then $E=E^{\mathrm{C}}$.

Figures 6(a) and 6(b) show the relationships between $E$ and $a(b=1)$ for different sets of $s$. Figure 6(a) shows the relationships for the case when the two constituent phases are of comparable rigidity, $E^{\mathrm{A}}=72.4 \mathrm{GPa}, E^{\mathrm{B}}=3.0 \mathrm{GPa}$. Figure $6(\mathrm{~b})$ shows the relationships for the case when phase $\mathrm{B}$ is almost negligible when compared with phase $\mathrm{A}, E^{\mathrm{A}}=3.0 \mathrm{GPa}, E^{\mathrm{B}}=100 \mathrm{~Pa}$. In these figures, the Voigt and Reuss mixing rules are also shown respectively,

$$
\begin{aligned}
& E=a E^{\mathrm{A}}+(1-a) E^{\mathrm{B}} \\
& E=\frac{E^{\mathrm{A}} E^{\mathrm{B}}}{(1-a) E^{\mathrm{A}}+a E^{\mathrm{B}}}
\end{aligned}
$$

where the Voigt rule corresponds to the case of $s=1$. 
As it can be seen from these figures, the Reuss mixing rule is effective for penalizing the intermediate value of $a$ when the Young's moduli are comparable in magnitude. It is not appropriate, however, when the magnitudes of constituent phases are of different scales. In contrast, the power function defined in Equations (36)-(41) is effective for both cases.

On the other hand, the Voigt mixing rule is applied for the Poisson's ratio $v$ as follows

$$
v=b\left\{a v^{\mathrm{A}}+(1-a) v^{\mathrm{B}}\right\}+(1-b)\left\{a v^{\mathrm{C}}+(1-a) v^{\mathrm{B}}\right\}
$$

where $v^{\mathrm{A}}, v^{\mathrm{B}}, v^{\mathrm{C}}$ are the Poisson's ratios of constituent phases A, B and C, respectively.

\subsection{Formulation of the topology optimization problem}

By using the aforementioned mixing rule, it is possible to analyse simultaneously, the topology of the macrostructure and the topology of the microstructure in theory, because this optimization problem is the extension of the method proposed by Bendsøe and Kikuchi [1]. However, it is impractical, since the number of the design variables becomes enormous. Therefore, in this paper, it is assumed that the structure consists of a single uniform composite material as shown in Figure 4. In this case, the number of design variables equals the number of finite elements of the unit cell for two-material-phase design, and the twice of that for three-material-phase design.

The design variables which prescribe the material phase of the $i$ th element are denoted by $a_{i}, b_{i}$, where $i=1,2, \ldots, N$, and $N$ denotes the number of the finite elements of the unit cell. The constraint conditions of this optimization problem are the volume fractions of the constituent materials:

$$
f_{\mathrm{A}}=\frac{\sum_{i=1}^{N} a_{i} b_{i}}{N}, \quad f_{\mathrm{B}}=\frac{\sum_{i=1}^{N}\left(1-a_{i}\right)}{N}, \quad f_{\mathrm{C}}=1-f_{\mathrm{A}}-f_{\mathrm{B}}
$$

where $f_{\mathrm{A}}, f_{\mathrm{B}}, f_{\mathrm{C}}$ are the volume fractions of constituent phases $\mathrm{A}-\mathrm{C}$. Here, it is assumed that the area of all finite elements of the unit cell is equal. In this case, the optimization problem defined by Equation (25) is rewritten as follows:

$$
\begin{gathered}
\min _{\boldsymbol{\alpha} \in \mathbf{L}}\left[C=\mathbf{d}^{\mathrm{T}} \mathbf{K}_{0} \mathbf{d}\right], \quad \boldsymbol{\alpha}=\left\{a_{1}, a_{2}, \ldots, a_{N}, b_{1}, b_{2}, \ldots, b_{N}\right\} \\
\mathbf{L}=\left\{\boldsymbol{\alpha} \mid f_{\mathrm{A}}^{\min } \leqslant f_{\mathrm{A}} \leqslant f_{\mathrm{A}}^{\max }, f_{\mathrm{B}}^{\min } \leqslant f_{\mathrm{B}} \leqslant f_{\mathrm{B}}^{\max }, 0 \leqslant a_{i} \leqslant 1,0 \leqslant b_{i} \leqslant 1, i=1, \ldots, N\right\}
\end{gathered}
$$

where $C$ denotes the mean compliance, $f^{\max }, f^{\text {min }}$ are the lower and upper limits of the volume fractions.

\subsection{Sequential linear programming method}

In this paper, the sequential linear programming method is used to solve the optimization problem defined by Equation (46). Figure 7 shows the flow chart of the optimization procedure. To begin with, the initial values of design variables are given. Next, the characteristic displacement function $\mathbf{X}$ is calculated from Equation (33), and $\mathbf{D}^{\mathrm{H}}$ is calculated by Equation (34). The mean compliance $C$ is obtained by solving Equation (23).

Next, it is necessary to calculate the sensitivities of the compliance and the volume fractions. The sensitivity of the mean compliance is calculated by the following equation:

$$
\frac{\partial C}{\partial \alpha_{i}}=-\mathbf{d}^{\mathrm{T}} \frac{\partial \mathbf{K}_{0}}{\partial \alpha_{i}} \mathbf{d}=-\sum_{\text {elements }} \mathbf{d}_{e}^{L^{\mathrm{T}}} \int_{D_{e}} \mathbf{B}_{0}^{\mathrm{T}} \frac{\partial \mathbf{D}^{\mathrm{H}}}{\partial \alpha_{i}} \mathbf{B}_{0} \mathrm{~d} D_{\mathrm{e}} \mathbf{d}_{\mathrm{e}}^{L}
$$


where $\alpha_{i}$ is the $i$ th component of $\boldsymbol{\alpha}$, other variables are already defined in Section 2, and the sensitivity of $\mathbf{D}^{\mathrm{H}}$ is calculated from Equation (34) as follows:

$$
\frac{\partial \mathbf{D}^{\mathrm{H}}}{\partial \alpha_{i}}=\int_{Y_{\mathrm{e}}}\left(\mathbf{I}-\mathbf{B}_{1} \mathbf{X}_{\mathrm{e}}\right)^{\mathrm{T}} \frac{\partial \mathbf{D}}{\partial \alpha_{i}}\left(\mathbf{I}-\mathbf{B}_{1} \mathbf{X}_{\mathrm{e}}\right) \mathrm{d} Y_{\mathrm{e}}
$$

The simplex method is used for solving the linear programming method, and the optimum solution of the increment value of $\boldsymbol{\alpha}$, viz. $\Delta \boldsymbol{\alpha}$, is obtained. Such calculation is repeated until all components of $\Delta \alpha$ are less than $\varepsilon_{\text {tol }}$.

The moving limit $\varepsilon$ is imposed in $\Delta \alpha$ as follows:

$$
-\varepsilon \leqslant \Delta \alpha_{i} \leqslant \varepsilon \quad(i=1, \ldots, 2 N)
$$

In this paper, the initial value of the moving limit is set to 0.5 , and we change the value by $\varepsilon / 1.05^{k}$ ( $k$ denotes the times of iteration) for $k \leqslant 50$, and $\varepsilon / 1.05^{50} / 1.1^{k-50}$ for $k>50$. And, the tolerance $\varepsilon_{\text {tol }}$ is set to 0.005 . In this case, the maximum number of iterations for optimization is 68 .

The constraints on the volume fraction of the constituent phases are applied in a step-wise fashion. It is difficult to find the global optimal solution if the volume fractions are set to the pre-defined bounds at the start of the optimization iteration. Therefore, for the relaxation we set these limit values as

$$
\begin{aligned}
f_{\mathrm{A}}^{\min } & =\bar{f}_{\mathrm{A}}\left[1-\left\{1-(k-1) / n_{c}\right\}\right] \\
f_{\mathrm{A}}^{\max } & =\bar{f}_{\mathrm{A}}\left[1+\lambda\left\{1-(k-1) / n_{c}\right\}\right], \quad\left(k \leqslant n_{c}+1\right) \\
f_{\mathrm{B}}^{\min } & =\bar{f}_{\mathrm{B}}\left[1-\left\{1-(k-1) / n_{c}\right\}\right] \\
f_{\mathrm{B}}^{\max } & =\bar{f}_{\mathrm{B}}\left[1+\lambda\left\{1-(k-1) / n_{c}\right\}\right], \quad\left(k \leqslant n_{c}+1\right) \\
f_{\mathrm{A}}^{\min } & =f_{\mathrm{A}}^{\max }=\bar{f}_{\mathrm{A}},\left(k>n_{c}+1\right) \\
f_{\mathrm{B}}^{\min } & =f_{\mathrm{B}}^{\max }=\bar{f}_{\mathrm{B}},\left(k>n_{c}+1\right) \\
\lambda & =\min \left[1 / \bar{f}_{\mathrm{A}}-1,1 / \bar{f}_{\mathrm{B}}-1,2\right]
\end{aligned}
$$

where $\bar{f}_{\mathrm{A}}, \bar{f}_{\mathrm{B}}, \bar{f}_{\mathrm{c}}$ are the volume fractions specified, $n_{c}$ denotes an appropriate number and $1 \leqslant n_{c} \leqslant n$ ( $n$ is the total number of iterations). In the numerical examples in this paper, we set $n_{c}$ to 30 .

\section{CHECKERBOARDING PROBLEM}

\subsection{Checkerboarding solutions}

In this paper, the four-node finite element proposed by Wilson and Tayler $[29,30]$ is used for the analysis of two-dimensional macrostructure, and the four-node isoparametric finite element is used for the analysis of microstructure. The checkerboarding solutions are obtained in the present approach, because the density approach with low order finite element is used for the analysis of the microstructure.

A numerical example is solved by the present approach in order to demonstrate this phenomenon. Figure 8 shows the example of a plate model with shear loads. The boundary condition, the loading condition and the finite element division of this model are shown in this figure. The thickness $t$ 


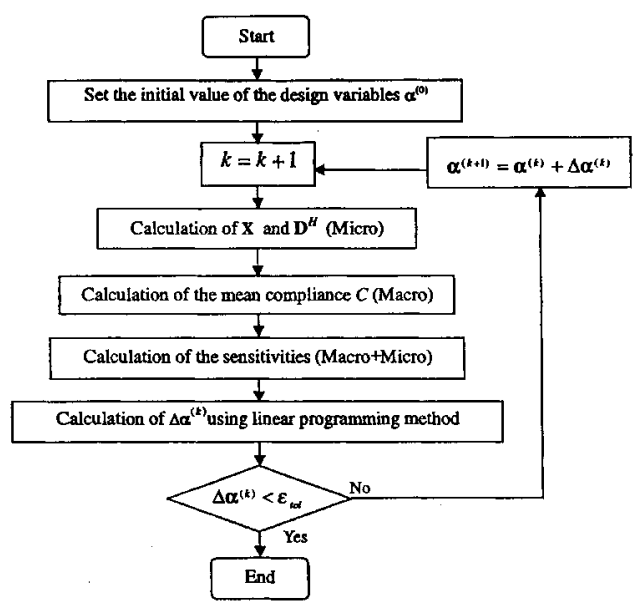

Figure 7. Flowchart of the optimization procedure.

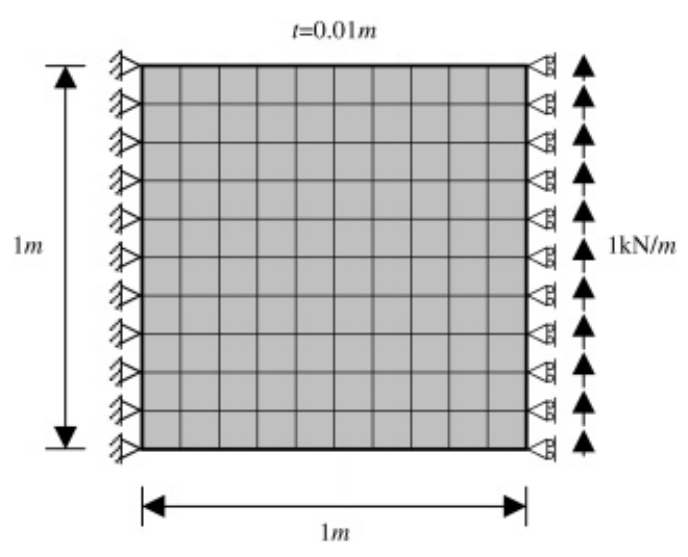

Figure 8. A plate model subjected to shear forces.

Table I. The properties of material phases.

\begin{tabular}{llcc}
\hline & & $E(\mathrm{GPa})$ & $v$ \\
\hline Grey material & Cast epoxy resin & 3.0 & 0.25 \\
Black material & E-glass fibre & 72.4 & 0.15 \\
White material & Void & $10^{-7}$ & 0.25 \\
\hline
\end{tabular}

of the plate is $0.01 \mathrm{~m}$. This problem is solved under the plane stress condition. In this analysis, the power $s$ in Equations (36)-(41) is set to 6 for the two-phase design and 5 for the three-phase design. The symmetrical condition with respect to the two axes $y_{1}, y_{2}$ and the diagonal line of the unit cell is imposed. The volume fractions of the constituent phases are set to $\bar{f}_{\mathrm{A}}=0.5$ and $\bar{f}_{\mathrm{B}}=0.5$ for two-phase materials, and $\bar{f}_{\mathrm{A}}=0.4, \bar{f}_{\mathrm{B}}=0.4$ and $\bar{f}_{\mathrm{C}}=0.2$ (void) for three-phase materials. We chose the E-glass fibre and resin epoxy used by Swan and Kosaka [10] as the two-component material phases. In the three-phase design problem, a very compliant material is used to simulate the void phase. The material properties each material phase are shown in Table I.

Figure 9 shows the solutions of the topology optimization of composite material of the plate for the two-phase design and the three-phase design, respectively. These figures show the distribution of the material phases in a unit cell, where the mesh division is $40 \times 40$. As shown in these figures, a lot of checkerboard patterns have appeared in these solutions.

\subsection{Perimeter control approach}

For the purpose of preventing such checkerboard solutions, several methods have been proposed by Haber et al. [24], Beckers [25], Duysinx [26], Swan and Kosaka [20], Petersson and Sigmund [27], and it was summarized by Sigmund and Petersson [21]. According to Sigmund and Petersson [21], the perimeter control approach [24-26] and the mesh-independent filtering approach [27], are effective to prevent such numerical instabilities in the topology optimization. 


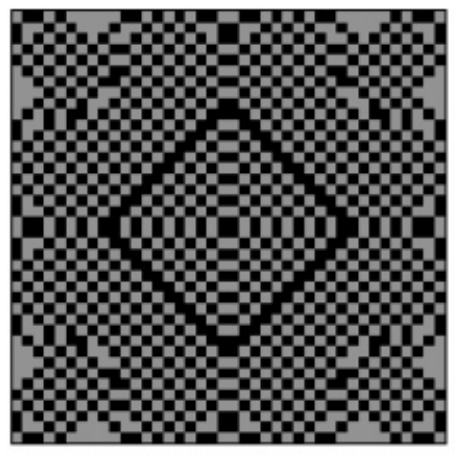

(a)

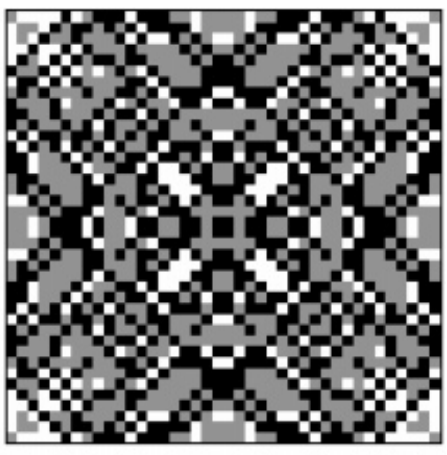

(b)

Figure 9. Optimal distribution of material phases in a unit cell for two- and three-phase designs:

(a) two-phase design; (b) three-phase design.

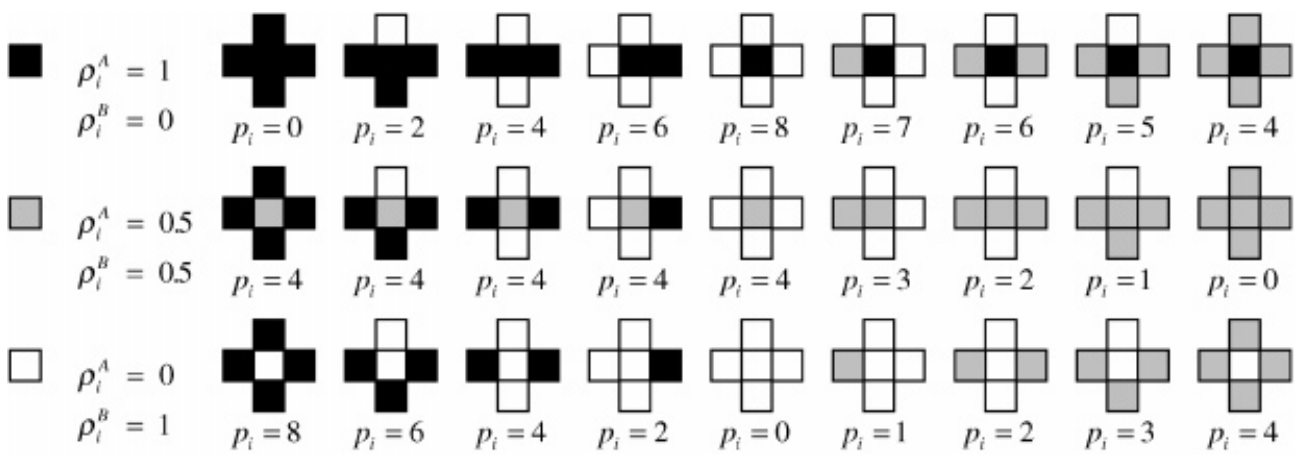

Figure 10. Values of the perimeter control function for some typical patterns of two-phase materials.

In the perimeter control approach, the checkerboarding solution is prevented by restricting the length of the perimeter which appears by the difference of the material density between adjacent elements. If the discretized perimeter control approach proposed by Beckers [25] is applied to the present approach, the perimeter control function $P$ is defined by

$$
P=\sum_{i=1}^{N} p_{i}, \quad p_{i}=\sum_{j=1}^{m_{i}} l_{i j}\left(\left|\rho_{i}^{\mathrm{A}}-\rho_{j}^{\mathrm{A}}\right|+\left|\rho_{i}^{\mathrm{B}}-\rho_{j}^{\mathrm{B}}\right|+\left|\rho_{i}^{\mathrm{C}}-\rho_{j}^{C}\right|\right)
$$

where $\rho_{i}^{\mathrm{A}}=a_{i} b_{i}, \rho_{i}^{\mathrm{B}}=1-a_{i}, \rho_{i}^{\mathrm{C}}=1-\rho_{i}^{\mathrm{A}}-\rho_{i}^{\mathrm{B}}$, and $m_{i}$ denotes the number of elements which have a common side with the $i$ th element $\left(m_{i}=4\right.$ for an interior elements, $m_{i}=3$ or 2 for edge or corner elements). Figure 10 shows the values of the perimeter control function $p_{i}$ for some typical patterns in the case of two-phase materials, where $m_{i}=4, l_{i j}=1$.

However, there are two problems in the perimeter control method. The first is that the setting of the constraint value of the perimeter length is difficult. If the constraint value of the perimeter length is too small, the convergent solution may not be obtained. If it is too large, the checkerboarding solution may occur. Many trials and errors are required in order to find the appropriate constraint 


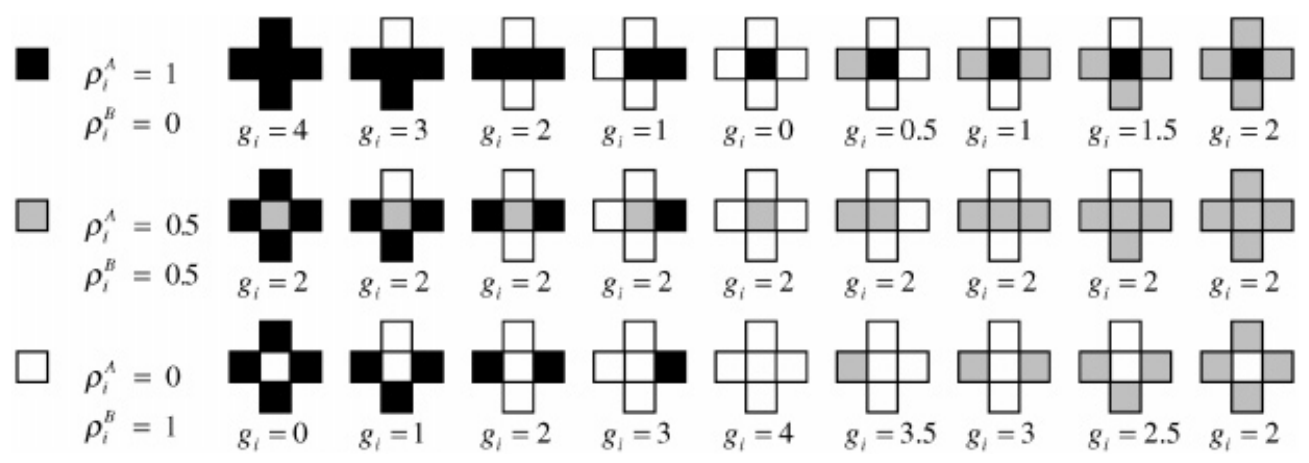

Figure 11. Values of the gravity function for some typical patterns of two-phase materials.

value. The second problem is that the mixed condition of the material phases is allowed in this method. It can be seen from figure that the pattern of 5 grey-scale elements yields the identical value of $p_{i}$ as the pattern of 5 black or white elements. That is to say, the penalty is not imposed on the grey-scale pattern. Therefore, if we use this method, the artificially mixed material phases are often not decomposed clearly in the solution. In the topology analysis of the macrostructure, the solution of the mixed phases may be admitted since they can be interpreted as a composite structure in the microscale. But in the analysis of the microstructure, the solution of the mixing phases is not favourable since one runs the risk of interpreting the 'microstructure of microstructure' in the regions of the mixed constituent phases.

\subsection{Proposed filter}

In this paper, a novel filtering method for addressing the shortcomings of the perimeter control method is proposed. In this method, the following gravity control function $G$ is used instead of the perimeter control function defined by Equation (51):

$$
G=\sum_{i=1}^{N} g_{i}, \quad g_{i}=\sum_{j=1}^{m_{i}}\left(\rho_{i}^{\mathrm{A}} \rho_{j}^{\mathrm{A}}+\rho_{i}^{\mathrm{B}} \rho_{j}^{\mathrm{B}}+\rho_{i}^{\mathrm{C}} \rho_{j}^{\mathrm{C}}\right)
$$

where $\rho_{i}^{\mathrm{A}}=a_{i} b_{i}, \rho_{i}^{\mathrm{B}}=1-a_{i}, \rho_{i}^{\mathrm{C}}=1-\rho_{i}^{\mathrm{A}}-\rho_{i}^{\mathrm{B}}$, and $m_{i}$ denotes the number of elements which have a common side with the $i$ th element. This function is based on the concept of gravity. Because the gravity force of the particles next to each other is defined by $\rho_{i} \rho_{j} / r_{i j}^{2}$, where $\rho_{i}, \rho_{j}$ are the masses of the particles, and $r_{i j}$ denotes the distance of the particles. When the size of the finite elements in the unit cell are all equal, the distances between the $i$ th element and the elements which have a common side with the $i$ th element are all equal. Therefore, in Equation (52), $r_{i j}^{2}$ has been removed. Figure 11 shows the values of the gravity control function $g_{i}$ for some typical patterns in the case of two-phase materials. It can be seen from Figure 11 that the penalty is imposed on the grey scale in this case, and the penalty is also imposed on the checkerboard pattern as well as the perimeter control method.

In this method, if the magnitude of the gravity control function increases, both the checkerboard and the grey-scale pattern decrease. Therefore, we add the gravity control function with a weighting ratio to the objective function as follows:

$$
f=C+w_{\mathrm{g}} \bar{C}\left(G / G_{\mathrm{ini}}\right)^{2}
$$




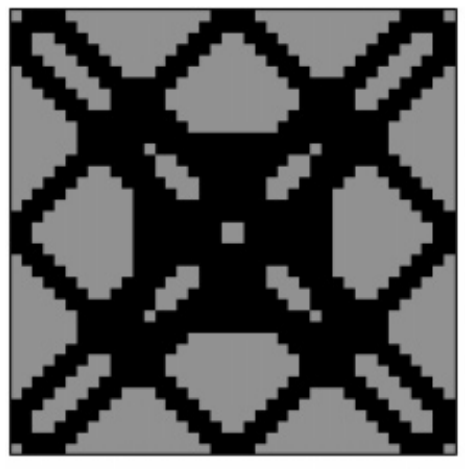

(a)

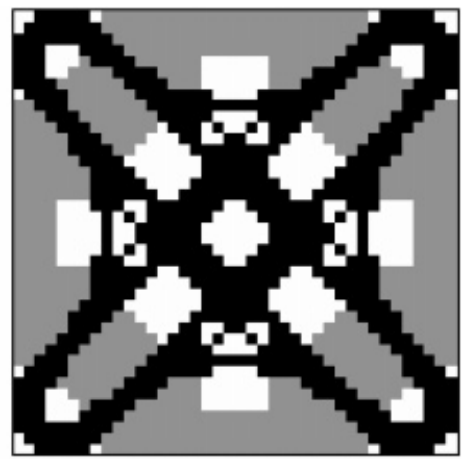

(b)

Figure 12. Optimal distribution of material phases in a unit cell for two- and three-phase designs:

(a) two-phase design; (b) three-phase design.

where $C$ and $\bar{C}$ are the mean compliance $(C=\bar{C}$ in each step), but $\bar{C}$ is just scaling constant and independent of the design variables (i.e. $\partial \bar{C} / \partial \alpha_{i}=0$ ). Another scaling constant, $G_{\text {ini }}$ is the value of the gravity function calculated at the initial iteration. The coefficient $w_{\mathrm{g}} \in[0,1]$ represents the weighting ratio between the mean compliance and the gravity function. The power coefficient $(=2)$ is set in order to emphasize the effect of the gravity control function. The sensitivity of this multi-objective function is calculated by

$$
\frac{\partial f}{\partial \alpha_{i}}=\frac{\partial C}{\partial \alpha_{i}}-2 w_{\mathrm{g}} \bar{C}\left(G / G_{i n i}^{2}\right) \frac{\partial G}{\partial \alpha_{i}}
$$

The numerical example shown in Figure 8 is solved to demonstrate the effectiveness of the proposed filtering method. Figure 12 shows the optimum topology solutions of the microstructure. The weighting ratios are set to 0.5 for two-phase design and 0.3 for three-phase design. It can be seen that clear topologies are obtained by using the proposed filtering method.

\section{DESIGN EXAMPLES}

In this section, some numerical examples are presented to demonstrate the applicability of the presented algorithm to the design of composite materials within the context of two-dimensional structures. We chose the E-glass fibre and resin epoxy used by Swan and Kosaka [5] as the twocomponent material phases. In the three-phase design problem, a very compliant material is used to simulate the void phase. The properties of these material phases are shown in Table I. In the following analysis, the power $s$ in Equations (36)-(41) is set to 6 for the two-phase design and 5 for the three-phase design. The volume fractions of the constituent phases are set to $\bar{f}_{\mathrm{A}}=0.5$ and $\overline{f_{\mathrm{B}}}=0.5$ for two-phase materials, and $\bar{f}_{\mathrm{A}}=0.4, \bar{f}_{\mathrm{B}}=0.4$ and $\overline{f_{\mathrm{C}}}=0.2$ (void) for three-phase materials. The mesh division of the unit cell is $40 \times 40$. These problems are solved under the plane stress condition.

Figure 13 shows the shear plate models which have different boundary conditions. The horizontal displacement of the right-hand edge is constrained in Case 1, and it is free in Case 2. The finite 


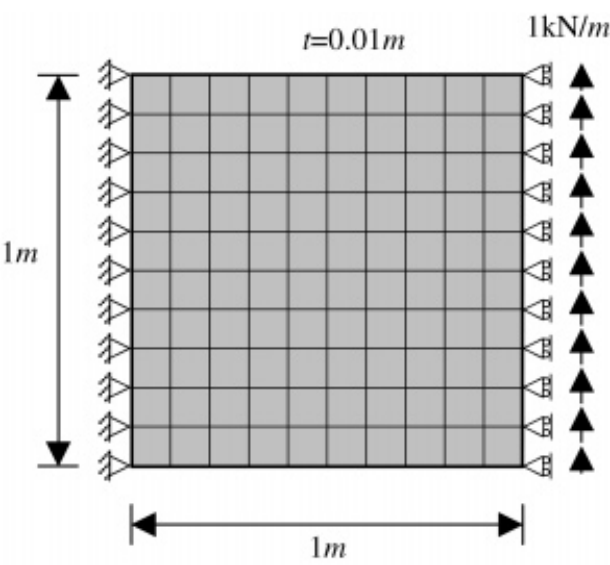

Case 1

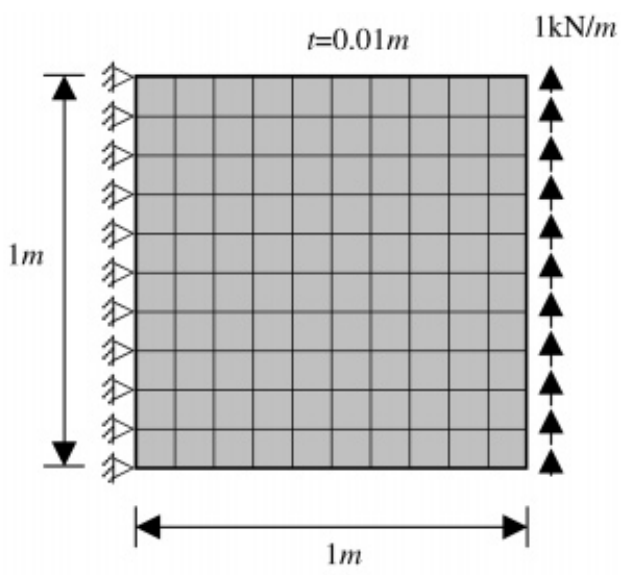

Case 2

Figure 13. Plate models subjected to shear forces with different boundary conditions.

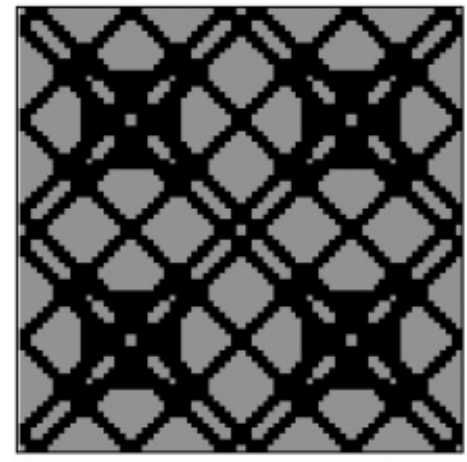

$w_{g}=0.5, C=1.72 \times 10^{-2}$

Case 1

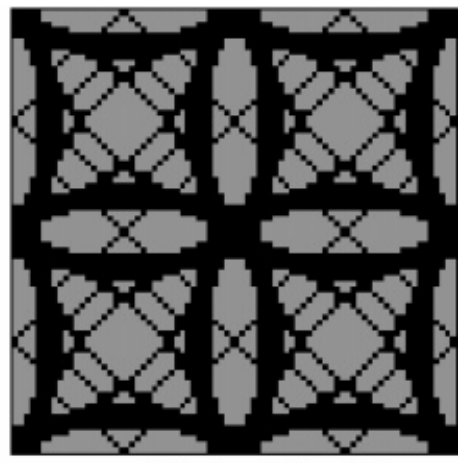

$w_{g}=0.5, C=3.52 \times 10^{-2}$

Case 2

Figure 14. Optimal distribution of material phases in four unit cells for two-phase design.

element division of the macrostructure is shown in these figures. The symmetrical condition with respect to the two axes $y_{1}, y_{2}$ and the diagonal line of the unit cell is imposed.

Figure 14 shows the solutions of the topology optimization of composite material for twomaterial-phase design. Figure 15 shows the solutions for three-material-phase design. These figures show the distribution of the material phase in four unit cells. The values of the mean compliance and the weighting ratio in Equation (53) are shown in these figures. Tables II and III show the components of the elasticity matrix in these solutions. It can be seen from these figures that the topology of Case 1 is clearly different from that of Case 2. Tables II and III show that the stiffness for the normal stresses of Case 2 is greater than that of Case 1. It also shows that the topology for two-phase design is similar to that of three-phase design, and the value of the shear stiffness $D_{33}^{\mathrm{H}}$ is greater than $9.5 \mathrm{GPa}$ which was obtained by Swan and Kosaka [5]. 

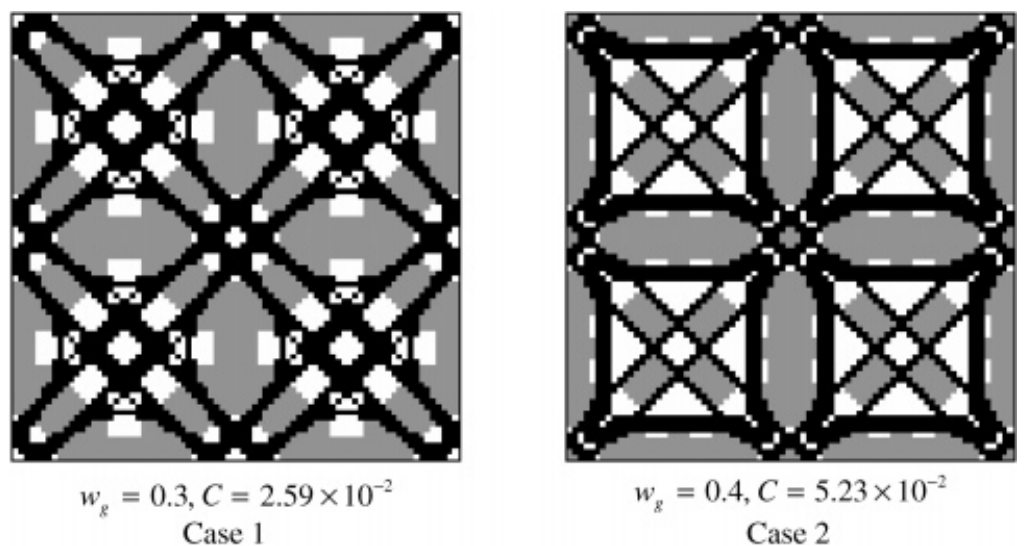

Figure 15. Optimal distribution of material phases in four unit cells for three-phase design.

Table II. Components of the elasticity matrix $D^{\mathrm{H}}$ for two-phase design.

\begin{tabular}{lccc}
\hline & $D_{11}^{\mathrm{H}}(\mathrm{GPa})$ & $D_{12}^{\mathrm{H}}(\mathrm{GPa})$ & $D_{33}^{\mathrm{H}}(\mathrm{GPa})$ \\
\hline Case 1 & 18.2 & 9.72 & 10.9 \\
Case 2 & 21.9 & 5.25 & 7.29 \\
\hline
\end{tabular}

Table III. Components of the elasticity matrix $D^{\mathrm{H}}$ for three-phase design.

\begin{tabular}{lccc}
\hline & $D_{11}^{\mathrm{H}}(\mathrm{GPa})$ & $D_{12}^{\mathrm{H}}(\mathrm{GPa})$ & $D_{33}^{\mathrm{H}}(\mathrm{GPa})$ \\
\hline Case 1 & 12.0 & 7.21 & 7.62 \\
Case 2 & 14.9 & 4.07 & 4.95 \\
\hline
\end{tabular}

Figure 16 shows the model of another design example. In this case, the loading condition is changed, and the boundary conditions are the same as the previous example. The symmetrical condition with respect to the two axes $y_{1}, y_{2}$ is also imposed, but the symmetrical condition with respect to the diagonal line is removed in this case. Figure 17 shows the solutions of the topology optimization of composite material for two-material-phase design. Figure 18 shows the solutions for three-material-phase design. Tables IV and V show the components of the elasticity matrix in these solutions. It can be seen from these figures that the topology of Case 1 is also different from Case 2. Table IV and V show that the stiffness in the $y_{2}$ direction is greater than that in the $y_{1}$ direction in Case 1, but the stiffness in the $y_{1}$ direction is greater than that in the $y_{2}$ direction in Case 2. It is also seen that the shear stiffness of Case 1 is greater than that of Case 2.

\section{CONCLUSIONS}

In this paper, the microstructural design algorithm of two-dimensional structures with composite materials was presented. Using this algorithm, it is possible to design both two- and three-phase microstructures. Using the artificial mixture assumption, the intermediate Young's modulus and 
$1 \mathrm{kN} / m$

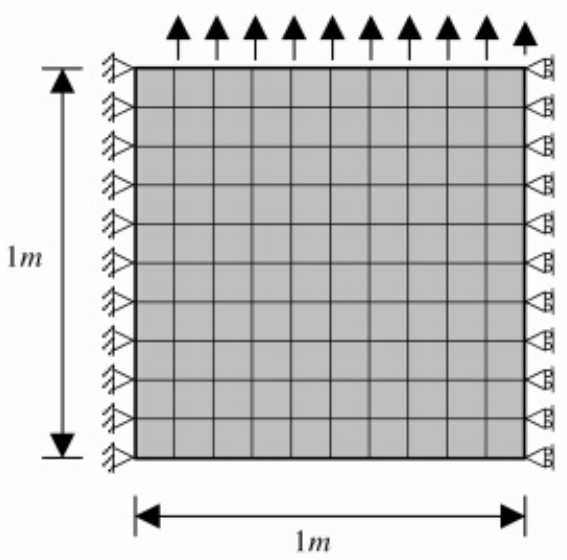

Case 1
$1 \mathrm{kN} / m$

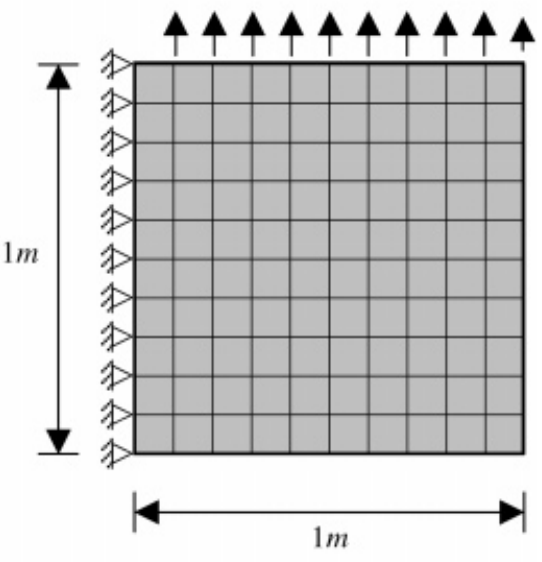

Case 2

Figure 16. Plate models subjected to normal forces with different boundary conditions.

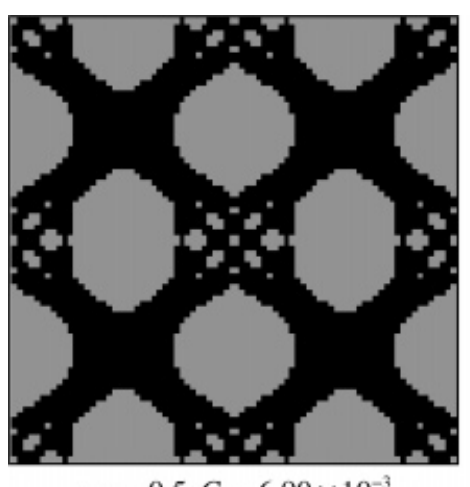

$w_{g}=0.5, C=6.90 \times 10^{-3}$

Case 1

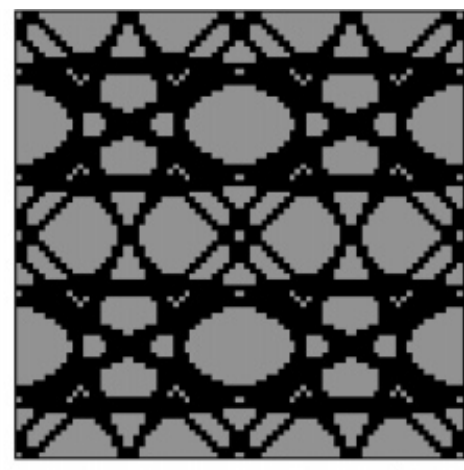

$$
w_{g}=0.6, C=9.19 \times 10^{-3}
$$

Case 2

Figure 17. Optimal distribution of material phases in four unit cells for two-phase design.

Poisson's ratio were made through the interpolation of two- or three-phase materials. The powerlaw function was used for the interpolation of the Young's modulus, and the linear function was used for the interpolation of the Poisson's ratio. The mean compliance of a macrostructure was chosen as the objective function, and the volume fractions of each material phase were given as the constraint conditions. To solve the optimization problem, the sequential linear programming method with move limit was used. A method for the relaxation of a fraction's constraint condition was used to prevent local minima. The filtering method based on the concept of gravity was used to prevent checkerboards. In this method, the gravity control function is added to the objective function with weight.

It was shown by the example of the shear plate that the proposed filtering method is effective for preventing the checkerboards. The effectiveness of the present algorithm for the design of composite 


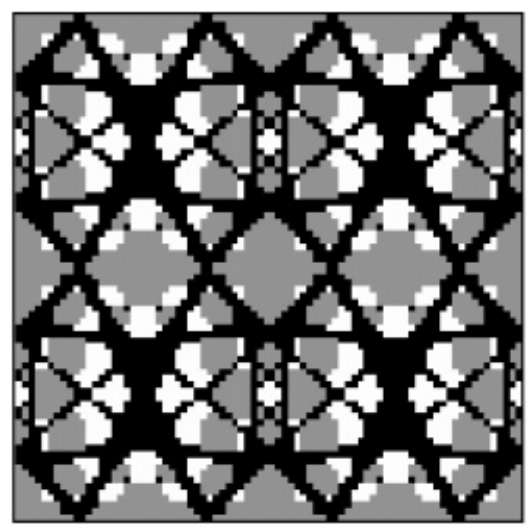

$w_{g}=0.5, C=1.09 \times 10^{-2}$

Case 1

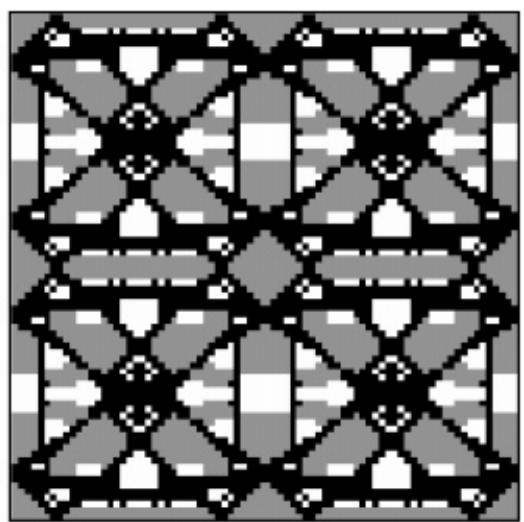

$$
w_{g}=0.5, C=1.50 \times 10^{-2}
$$

Case 2

Figure 18. Optimal distribution of material phases in four unit cells for three-phase design.

Table IV. Components of the elasticity matrix $D^{\mathrm{H}}$ for two-phase design.

\begin{tabular}{lcccc}
\hline & $D_{11}^{\mathrm{H}}(\mathrm{GPa})$ & $D_{12}^{\mathrm{H}}(\mathrm{GPa})$ & $D_{22}^{\mathrm{H}}(\mathrm{GPa})$ & $D_{33}^{\mathrm{H}}(\mathrm{GPa})$ \\
\hline Case 1 & 17.9 & 9.01 & 19.9 & 10.1 \\
Case 2 & 25.5 & 5.92 & 18.5 & 8.15 \\
\hline
\end{tabular}

Table V. Components of the elasticity matrix $D^{\mathrm{H}}$ for three-phase design.

\begin{tabular}{lcccc}
\hline & $D_{11}^{\mathrm{H}}(\mathrm{GPa})$ & $D_{12}^{\mathrm{H}}(\mathrm{GPa})$ & $D_{22}^{\mathrm{H}}(\mathrm{GPa})$ & $D_{33}^{\mathrm{H}}(\mathrm{GPa})$ \\
\hline Case 1 & 12.5 & 4.78 & 14.3 & 5.72 \\
Case 2 & 16.4 & 3.87 & 11.9 & 4.82 \\
\hline
\end{tabular}

material was demonstrated by some numerical examples which have different boundary or loading conditions. That is to say, the distinctive topology of the microstructures of the composite material can be obtained using the present algorithm. The efficiency of the calculation of the present algorithm was also verified because the number of iterations required for the optimization procedure was less than 68.

Finally, we note that the algorithm presented here can be directly extended to three-dimensional problems.

\section{ACKNOWLEDGEMENTS}

This research was partially supported by Steel Material Club Corporation Aggregate in Japan. Its support is gratefully acknowledged.

\section{REFERENCES}

1. Bendsøe MP, Kikuchi N. Generating optimal topologies in structural design using a homogenization method. Computer Methods in Applied Mechanics and Engineering 1988; 71:197-224. 
2. Suzuki K, Kikuchi N. A homogenization method for shape and topology optimization. Computer Methods in Applied Mechanics and Engineering 1991; 93:291-318.

3. Murat F, Tartar L. Calcul des variations et homogeneisation, in: les methodes de l'homogeneisation: theorie et applications en physique. Coll. de la Dir. des Etudes et Recherches de Electricite de France. Eyrolles, Paris, 1985; 319-370.

4. Sigmund O. Material with prescribed constitutive parameters: an inverse homogenization problem. International Journal of Solids and Structures 1994; 31(17):2313-2329.

5. Sigmund O. Tailoring materials with prescribed elastic properties. Mechanics of Materials 1995; 20(4):351-368.

6. Sigmund O. Design of material structures using topology optimization. Report No. 502, Danish Center for Applied Mathematics and Mechanics, Technical University of Denmark, 1995; 43-50.

7. Sigmund O, Torquato S. Design of materials with extreme thermal expansion using a three-phase topology optimization method. Journal of the Mechanics and Physics of Solids 1997; 45(6):1037-1067.

8. Fonseca JSO. Design of microstructures of periodic composite materials. PhD Thesis, The University of Michigan, 1997.

9. Swan CC, Arora JS. Topology design of material layout in structured composites of high stiffness and strength. Structural Optimization 1997; 13:45-59.

10. Swan CC, Kosaka I. Homogenization-based analysis and design of composites. Computers and Structures 1997; 64:603-621.

11. Silva, ECN, Kikuchi N. Optimal design of piezoelectric microstructures. Computer Mechanics 1997; 19(5):398-410.

12. Silva ECN, Fonseca JSO, Kikuchi N. Optimal design of periodic piezocomposites. Computer Methods in Applied Mechanics and Engineering 1998; 159:49-77.

13. Gibiansky LV, Torquato S. On the use of homogenization theory to design optimal piezocomposites for hydrophone applications. Journal of the Mechanics and Physics of Solids 1997; 45(5):689-708.

14. Gibiansky LV, Torquato S. Optimal design of 1-3 composite piezoelectrics. Structural Optimization 1997; 13:23-28.

15. Haslinger J, Dvorak J. Optimum composite material design. RAIRO Modelisation Mathematiqueet Analyse Numerique 1995; 29(6):657-686.

16. Bendsøe MP. Optimal shape design as a material distribution problem. Structural Optimization 1989; 1:193-202.

17. Rozvany GIN, Zhou M, Birker T, Sigmund O. Topology optimization using iterative continuum type optimality criteria (COC) methods for discretized systemes. In Topology Design of Structures. Bendsøe MP, Soares CA (eds), Springer: Berlin, 1992; 273-286.

18. Mlejnek HP, Schirrmacher R. An engineer's approach to optimal material distribution and shape finding. Computer Methods in Applied Mechanics and Engineering 1993; 106:1-26.

19. Yang RJ, Chuang CH. Optimal topology design using linear programming. Computers and Structures 1994; 52: $265-275$.

20. Swan, CC, Kosaka I. Voigt-Reuss topology optimization for structures with linear elastic material behaviors. International Journal for Numerical Methods in Engineering 1997; 40:3033-3057.

21. Sigmund O, Petersson J. Numerical instabilities in topology optimization: A survey on procedures dealing with checkerboards, mesh-dependencies and local minima. Structural Optimization 1998; 16:68-75.

22. Jog CS, Habar RB. Stability of finite element models for distributed-parameter optimization and topology design. Computer Methods in Applied Mechanics and Engineering 1996; 130:203-226.

23. Diaz A, Sigmund O. Checkerboard patterns in layout optimization. Structural Optimization 1995; 10:40-45.

24. Haber RB, Bendsøe MP, Jog C. A new approach to variable-topology shape design using a constraint on the perimeter. Structural Optimization 1996; 11:1-12.

25. Beckers M. Optimisation de structures en variables disct3tes. Th3se de Doctorat, Universite de Liege, Ing3nieur civil 3lectricien-m3canicien(a3rospatiale), 1997; 108-135.

26. Duysinx P. Layout optimization: a mathematical programming approach. DACAMM Report, No.540, Technical University of Denmark, 1997.

27. Petersson J, Sigmund O. Slope constrained topology optimization. International Journal for Numerical Methods in Engineering 1998; 41:1417-1434.

28. Lions JL, Some Methods in the Mathematical Analysis of Systems and Their Control. Science Press: Beijing, China, Gordon and Breach Science Publishers: New York, 1981.

29. Wilson EL, Taylor RL, Doherty WP, Ghaboussi J. Incompatible displacement models. In Numerical and Computer Methods in Structural Mechanics, Fenves ST et al. (eds), Academic Press: New York, 1973; 43-57.

30. Taylor RL, Beresford PJ, Wilson EL. A non-conforming element for stress analysis. International Journal for Numerical Methods in Engineering 1976; 10:1211-1220. 